\title{
Reply to: Is presymptomatic spread a major contributor to COVID-19 transmission?
}

\author{
Eric H. Y. Lau $₫ \bowtie$ and Gabriel M. Leung $₫$
}

REPLYING TO M. K. Slifka \& L. Gao Nature Medicine https://doi.org/10.1038/s41591-020-1046-6 (2020)

Slifka and $\mathrm{Gao}^{1}$ questioned the plausibility and extent of presymptomatic transmission of severe acute respiratory syndrome coronavirus 2 (SARS-CoV-2), in part in relation to our recent report ${ }^{2}$. We provide our response below.

Our study utilized two sources-a clinical dataset with viral load data from 94 patients with coronavirus disease 2019 (COVID-19) and an epidemiologic dataset with 77 infector-infectee transmission pairs. The estimated infectiousness profile, including the proportion of presymptomatic transmission, was based on the latter dataset alone, which we had made clear in our original report. Slifka and Gao misunderstood our argument that the viral load data or their trend was used to infer the temporal infectiousness profile. We specifically did not and could not adopt the said approach because of data limitations worldwide at the time of publication. For example, there were only five throat swabs in total during the first three days after symptom onset in two early studies with longitudinal viral load data, one of which Slifka and Gao cited ${ }^{3,4}$. In any case, viral load data obtained by reverse-transcription PCR do not reflect virus viability and should not be used directly or solely to infer infectiousness. Fitting a longitudinal statistical model or analysis of individual peak viral loads would not resolve this fundamental data limitation. As such, we used a cubic spline only for the purpose of data visualization. We presented the viral load data to provide context vis-à-vis the plausibility of the main findings on the infectiousness profile derived from infector-infectee transmission pairs. We were not unaware of this potential misinterpretation; thus, in the main text we explicitly stated that "viral load data were not used in the estimation but showed a similar monotonic decreasing pattern" ${ }^{2}$.

Slifka and Gao used a different (lognormal) incubation period with mean and standard deviation parameters of 1.57 and 0.65 from $\mathrm{Bi}$ et $\mathrm{al}^{5}{ }^{5}$ (that is, median $=4.80$ days and mean $=5.95$ days) to estimate the infectiousness profile with a (shifted) gamma distribution, and estimated that infectiousness peaked at 2.3 days before symptom onset, which is implausible as shown in the bottom right panel of Fig. 1 in Slifka and Gao. The peak might have been obtained by overlooking that the mode is the same as the start of infectiousness when the shape parameter is smaller than 1 for a gamma distribution. We refitted the model using the same incubation period and obtained parameters with a larger log-likelihood value $(-189.48$ versus -189.63 from the original code, which was not yet optimized for the alternative incubation period), and estimated that infectiousness started 2.5 days and peaked at 1.0 day before symptom onset (Fig. 1). The estimated proportion of presymptomatic spread was $57 \%$. This larger presymptomatic proportion could have been anticipated with a longer incubation period (mean $=5.2$ days in our study).
Since the publication of our paper and Slifka and Gao's correspondence, we have identified a syntactical error in the original code. Using a mean incubation period of 5.2 days, the amended estimation gave a presymptomatic proportion of $44 \%$ (unchanged from the original estimate). This is now detailed in a correction ${ }^{2}$.

Further, Slifka and Gao cited the report of $\mathrm{Du}$ et al. ${ }^{6}$, wherein $12.6 \%$ of the serial intervals were negative, as an indication of the presymptomatic proportion. While a negative serial interval precludes the possibility of transmission after symptom onset of the infector, a non-negative serial interval does not rule out presymptomatic transmission. For example, a serial interval of 0 indicates a $50 \%$ chance of presymptomatic transmission if we assume the incubation periods of the infector and infectee were similar. Therefore, the $12.6 \%$ negative serial interval datum provides only a very conservative lower bound for the presymptomatic proportion. Our modeling has accounted for such to estimate the likely, not minimum, proportion of presymptomatic spread.

Slifka and Gao also cited contrarian studies with selective, and perhaps biased, samples. For example, one study from Nanjing looked at 24 individuals with asymptomatic COVID-19 who were close contacts of confirmed cases? . Some of these patients were admitted and individually isolated in hospital as early as two days before symptom onset, which would likely have prevented further transmission, in the community or nosocomially. Similarly, an outbreak at a South Korean call center involving almost 100 confirmed cases was cited, where early and stringent quarantine and isolation measures had been implemented after the first case was reported $^{8}$. Patients in another Singaporean study were identified through contact tracing that considered only contacts after symptom onset of the infector, thus limiting the possibility of identifying presymptomatic transmission ${ }^{9}$. In fact, the authors of that study strongly recommended that contact tracing should include a period before symptom onset. Slifka and Gao also misinterpreted 55\% as the proportion of transmission due to undocumented cases in $\mathrm{Li}$ et al. ${ }^{10}$, as opposed to the relative transmissibility of undocumented cases, which they correctly differentiated from presymptomatic cases. Slifka and Gao also incompletely referenced an observational study wherein $31 \%(5 / 16)$ of transmission occurred before symptom onset. In fact, these five transmission events occurred exactly one day before symptom onset, but four more transmission events occurred two to three days before symptom onset (Fig. 2C in Furuse et al. ${ }^{11}$ ). This suggested a substantial 56\% (9/16) chance of presymptomatic transmission, which is consistent with our results.

Slifka and Gao based some of their arguments on the comparative transmissibility of SARS-CoV-2 with Middle East respiratory

World Health Organization Collaborating Centre for Infectious Disease Epidemiology and Control, School of Public Health, University of Hong Kong,

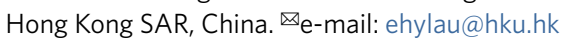




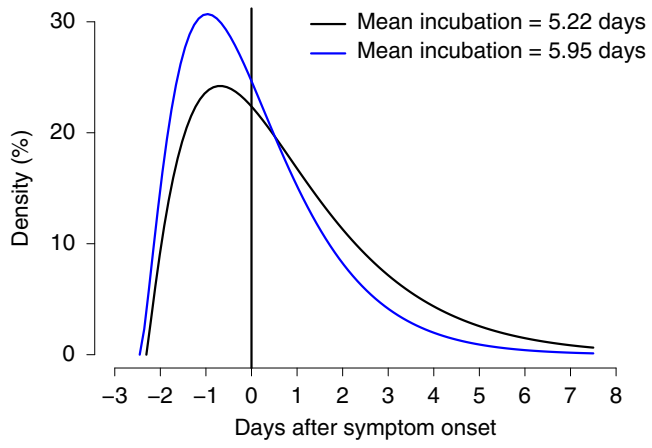

Fig. 1 | Inferred infectiousness profiles. Estimation was based on mean incubation periods of 5.22 days and 5.95 days (Bi et al. ${ }^{5}$ ), respectively.

syndrome coronavirus and SARS-CoV-1, which had very different viral kinetics and shedding profiles, respectively ${ }^{12,13}$.

For SARS-CoV-2, a recent study investigating contact tracing strategies estimated the proportion of presymptomatic transmission at $35 \%$, similar to our estimate. It also found that other modes of transmission (symptomatic, environmental and asymptomatic) contribute less than presymptomatic spread.

Allow us to re-emphasize what we had fully acknowledged previously, that our estimated infectiousness profile was obtained "in settings with substantial household clustering, active case finding and quarantine outside the home". Such interventions would have a stronger effect of reducing transmission after symptom onset, thus shifting the infectiousness profile distribution towards the presymptomatic period.

We conclude that presymptomatic transmission of SAR-CoV-2 is not only plausible, but quite possibly a substantial mode of transmission, especially in the presence of public health interventions.

\section{Online content}

Any methods, additional references, Nature Research reporting summaries, source data, extended data, supplementary information, acknowledgements, peer review information; details of author contributions and competing interests; and statements of data and code availability are available at https://doi.org/10.1038/ s41591-020-1049-3.

Received: 19 June 2020; Accepted: 3 August 2020; Published online: 17 August 2020

\section{References}

1. Slifka, M. K. \& Gao, L. Is presymptomatic spread a major contributor to COVID-19 transmission? Nat. Med. https://doi.org/10.1038/s41591-0201046-6 (2020).

2. He, X. et al. Temporal dynamics in viral shedding and transmissibility of COVID-19. Nat. Med. 26, 672-675 (2020); author correction https://doi. org/10.1038/s41591-020-1016-z (2020).

3. Wolfel, R. et al. Virological assessment of hospitalized patients with COVID-2019. Nature 581, 465-469 (2020).

4. Zou, L. et al. SARS-CoV-2 viral load in upper respiratory specimens of infected patients. N. Engl. J. Med. 382, 1177-1179 (2020).

5. Bi, Q. et al. Epidemiology and transmission of COVID-19 in 391 cases and 1286 of their close contacts in Shenzhen, China: a retrospective cohort study. Lancet Infect. Dis. 20, 911-919 (2020).

6. Du, Z., Xu, X., Wu, Y., Wang, L., Cowling, B. J. \& Meyers, L. A. Serial interval of COVID-19 among publicly reported confirmed cases. Emerg. Infect. Dis. 26, 1341-1343 (2020).

7. $\mathrm{Hu}, \mathrm{Z}$. et al. Clinical characteristics of 24 asymptomatic infections with COVID-19 screened among close contacts in Nanjing, China. Sci. China Life Sci. 63, 706-711 (2020).

8. Park, S. Y. et al. Coronavirus disease outbreak in call center, South Korea. Emerg. Infect. Dis. 26, 1666-1670 (2020).

9. Wei, W. E. et al. Presymptomatic transmission of SARS-CoV-2 - Singapore, January 23-March 16, 2020. MMWR Morb. Mortal. Wkly. Rep. 69, 411-415 (2020).

10. Li, R. et al. Substantial undocumented infection facilitates the rapid dissemination of novel coronavirus (SARS-CoV-2). Science 368, 489-493 (2020).

11. Furuse, Y. et al. Clusters of coronavirus disease in communities, Japan, January-April 2020. Emerg. Infect. Dis. https://doi.org/10.3201/eid2609.202272 (2020).

12. To, K. K. et al. Temporal profiles of viral load in posterior oropharyngeal saliva samples and serum antibody responses during infection by SARS-CoV-2: an observational cohort study. Lancet Infect. Dis. 20, 565-574 (2020).

13. Peiris, J. S. et al. Clinical progression and viral load in a community outbreak of coronavirus-associated SARS pneumonia: a prospective study. Lancet 361, 1767-1772 (2003).

(c) The Author(s), under exclusive licence to Springer Nature America, Inc. 2020 


\section{Data availability}

Detailed transmission-pair data in this study are provided in the Supplementary Information of the original study.

\section{Code availability}

The code for generating Fig. 1 can be found in the Supplementary Information and at https://github.com/ehylau/COVID-19.

\section{Author contributions}

E.H.Y.L. performed the additional analysis. E.H.Y.L. and G.M.L. wrote the manuscript.

\section{Competing interests}

The authors declare no competing interests.

\section{Additional information}

Supplementary information is available for this paper at https://doi.org/10.1038/ s41591-020-1049-3.

Correspondence and requests for materials should be addressed to E.H.Y.L.

Peer review information Joao Monteiro was the primary editor on this article and managed its editorial process and peer review in collaboration with the rest of the editorial team.

Reprints and permissions information is available at www.nature.com/reprints. 